Revue internationale de l'économie sociale

Recma

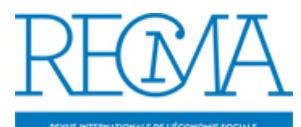

\title{
En bref
}

Numéro 313, juillet 2009

URI : https://id.erudit.org/iderudit/1020919ar

DOI : https://doi.org/10.7202/1020919ar

Aller au sommaire du numéro

\section{Éditeur(s)}

Association Recma

\section{ISSN}

1626-1682 (imprimé)

2261-2599 (numérique)

Découvrir la revue

Citer ce document

(2009). En bref. Revue internationale de l'économie sociale, (313), 14-17.

https://doi.org/10.7202/1020919ar d'utilisation que vous pouvez consulter en ligne.

https://apropos.erudit.org/fr/usagers/politique-dutilisation/ 


\section{EN BREF*}

\section{L'économie sociale}

\section{Quelques eurodéputés proches de l'ES}

Sur les soixante-douze députés français élus le 7 juin dernier au Parlement européen, sept sont convaincus de la nécessité d'y défendre l'économie sociale ou s'y sont déclarés sensibles lors de rencontres organisées par le Ceges et ses membres: Pervenche Bérès (PS), Jean-Marie Cavada (NC), Liem Hoang-Ngoc (PS), Sylvie Goulard (Modem) et Jean-Paul Gauzes (UMP), Joseph Daul (UMP), Marie-Christine Vergiat (DVG) et Pascal Canfin (DV). Espérons que l'intergroupe économie sociale au Parlement s'en trouve renforcé. Pour en savoir plus: www.ceges.org.

\section{Les Cres ou comment faire beaucoup avec peu de moyens}

Le Conseil national des chambres régionales de l'économie sociale (CNCres) vient de publier un panorama des vingt-trois $\mathrm{Cres}(\mathrm{s})$ existantes. Quinze sont des chambres de l'économie sociale, huit de l'économie sociale et solidaire (dont trois, la Guadeloupe, la Corse et la Guyane, ont rejoint le réseau fin 2008-début 2009). Le document précise leur structuration (nombre et nature des collèges, des adhérents...) et la composition de leurs instances dirigeantes. On notera ainsi que $31 \%$ des présidents représentent le monde associatif, $26 \%$ sont issus de la mutualité de santé (13\% de l'assurance), $17 \%$ de la coopération et $4 \%$ de l'économie solidaire. Le panorama détaille ensuite les moyens financiers et humains dont disposent les Cres. Trois seulement comptent plus de dix ETP (Rhône-Alpes, Aquitaine et Bretagne). Le réseau embauche en tout 124 personnes (plus de $80 \%$ en CDI) pour 118 ETP,

* Retrouvez les actualités, informez-nous ou contribuez directement sur http://recma.org. pour un budget moyen de 368700 euros, mais avec de fortes disparités (de 40100 à 1,29 million d'euros), soit 8,5 millions d'euros en tout (les principaux financeurs étant la région, le FSE et la DIIESES). Autrement dit, de tous petits moyens au regard de l'impressionnante liste de missions et de productions réalisées par les Cres depuis 2006!

Pour en savoir plus: www.cncres.org.

\section{Le prix Edgard Milhaud du Ciriec}

Le conseil international du Centre international de recherches et d'information sur l'économie publique, sociale et coopérative (Ciriec), qui compte quinze sections nationales, est, dans lintervalle des assemblées générales, l'organe de direction de l'organisation. Il s'est réuni à Lisbonne le 27 mars dernier, à l'invitation de la section portugaise, et a décidé la création d'un prix Edgard Milhaud, du nom du fondateur du Ciriec, destiné à " récompenser le travail d'un jeune chercheur ou expert sur une question d'intérêt pour le Ciriec liée au thème de ses congrès biannuels». D'un montant de 10000 euros, il sera remis tous les deux ans, à l'occasion des congrès internationaux du Ciriec. Les candidats seront présentés par les sections nationales ou des membres du Ciriec. Pour en savoir plus: www.ciriec.ulg.ac.be.

\section{Gérard Andreck à la présidence du Ceges}

A l'issue de l'assemblée générale et du conseil d'administration du Ceges du 12 mai, Gérard Andreck, président du Groupement des entreprises mutuelles d'assurances (Gema), président de la Macif, a été porté à la présidence du Conseil des entreprises, employeurs et groupements de l'économie sociale. Il succède à JeanClaude Detilleux, président du Groupement national de la coopération (GNC), président du Crédit coopératif, qui occupait cette fonction depuis 2005. Avec Gérard Andreck, la 
famille des mutuelles d'assurances relaie la famille coopérative dans cette responsabilité de promouvoir l'économie sociale et de la faire reconnaittre comme un acteur économique à part entière. Pour en savoir plus: www.ceges.org.

\section{L'ESS en région:}

\section{le site des politiques publiques}

L'Association des régions de France (ARF) s'intéresse de près à l'ESS (voir la présentation de son Manifeste pour une économie sociale et solidaire présenté dans le numéro 311 de la Recma). Avec l'Avise et la Caisse des dépôts, elle vient de mettre en ligne un nouveau site présenté comme un centre de ressources sur l'ESS dans les territoires. Il vise à présenter les politiques régionales de soutien au secteur, afin de valoriser les dispositifs et les pratiques innovants et d'offrir un espace de mutualisation et d'échange aux techniciens, ainsi qu'aux élus des conseils régionaux et aux chargés de mission des directions régionales des caisses des dépôts.

Pour en savoir plus: www.essenregion.org.

\section{La coopération}

\section{Un nouveau président à la FFCGA...}

Pascal Madec, artisan plombier du Finistère, a été élu à la présidence Fédération française des coopératives et groupements d'artisans (FFCGA) lors de l'assemblée générale qui s'est tenue le 27 mai. Il succède à Hubert Libotte, qui présidait la FFCGA depuis 2006. Ce dernier a reçu la médaille de la reconnaissance artisanale des mains d'Alain Griset, président de l'Assemblée permanente des chambres des métiers, qui marque ainsi son attachement à la coopération. La FFCGA fêtait alors ses trente ans en organisant les Assises de la coopération. Les autorités de l'Etat (DIIESES, DGCIS) et le président de l'Union professionnelle artisanale ont conclu cette journée.

Pour en savoir plus: www.ffcga.coop.

\section{... à la présidence de la Fédération nationale des Caisses d'épargne...}

Deux jours plus tôt, Michel Sorbier était élu pour six ans à la présidence de la FNCE, organe de représentation et d'expression des Caisses d'épargne et de leurs sociétaires. Il succède à Nicole Moreau, présidente depuis 1999. Cette élection clôt le processus de renouvellement de la gouvernance coopérative au sein du réseau, les assemblées générales électives de sociétaires s'étant tenues en janvier dans toutes les Caisses d'épargne, de nouveaux conseils d'orientation et de surveillance ayant été également mis en place. Michel Sorbier a été agent général d'assurances à Limoges durant toute sa carrière professionnelle, jusqu'en 2007. Il a présidé la Caisse d'épargne du Limousin de février 1992 à avril 2003, puis celle d'Auvergne et du Limousin, à laquelle il a été réélu le 9 avril dernier. Au niveau local, Michel Sorbier est président de la Société locale d'épargne de Limoges ville. Il est membre du conseil d'administration de la Fédération nationale des Caisses d'épargne depuis 1999.

Pour en savoir plus: www.fnce.fr.

\section{... et au Crédit coopératif !}

Le conseil d'administration du Crédit coopératif du 28 mai 2009 a porté Jean-Louis Bancel à la présidence du groupe. Vice-président délégué depuis janvier 2005, il succède à Jean-Claude Detilleux, en charge de la présidence depuis 1992. Jean-Louis Bancel siège au conseil d'administration du Crédit coopératif depuis 2000, où il a représenté la Mutualité française jusqu'en 2004. Il a présidé, en 1999-2000, le conseil national du Crédit coopératif, instance qui regroupe les représentants des sociétaires et partenaires du Groupe Crédit coopératif. Il est membre du comité des risques du conseil d'administration. Il est administrateur de la Banque fédérale des Banques populaires depuis mai 2009. Il préside l'Association internationale des banques coopératives (AIBC), composante de l'Alliance coopérative internationale (ACI). Il est membre du collège du Conseil national de la comptabilité. A la demande de Jean-Louis Bancel, le conseil 
d'administration a confié à Jean-Claude Detilleux un mandat de vice-président délégué chargé du rayonnement des valeurs coopératives. Pour en savoir plus: www.credit-cooperatif.coop.

\section{Les coopératives de santé dans le monde}

L'Institut de recherche et d'éducation pour les coopératives et les mutuelles de l'université de Sherbrooke (Irecus) vient de publier en libre accès sur son site, avec le concours notamment des Caisses Desjardins, de l'Organisation internationale des coopératives de santé (IHCO) et du secrétariat aux Coopératives du gouvernement du Canada, cinq études nationales sur la place des coopératives dans des systèmes de santé fort différents: Bénin, Mali, Canada, Ouganda et Etats-Unis, où Group Health Co-op est souvent cité comme modèle pouvant inspirer un système universel d'assurance maladie. Une base de comparaison riche d'enseignements qui devrait bientôt déboucher sur un document de synthèse Pour en savoir plus: www.usherbrooke.cal irecus/centre_documentation/coop_sante_fr.html.

\section{Les associations}

\section{Le point sur le projet Waldec}

Lancé en 2001, le projet « Web des associations librement déclarées " (Waldec) a bien avancé. Il ambitionne de répondre aux besoins exprimés par les administrations et par le monde associatif en permettant l'échange électronique des données de déclaration ainsi que la dématérialisation des procédures de déclaration. Mieux encore, le projet Waldec «devait également comprendre un programme d'analyse simplifié des comptes des associations", selon le Haut Commissariat aux solidarités actives et à la jeunesse. De quoi aider bientôt à la production de chiffres de référence sur les associations. Près de 500000 d'entre elles sont déjà entrées dans les bases de données à l'usage réservé des services de l'Etat et 1,7 million de dossiers (y compris donc les associations dissoutes) ont été recopiés. Cinq départements sont encore à équiper.

Question n 37574, réponse du 26 mai 2009 sur http://questions.assemblee-nationale.fr.

\section{Les mutuelles}

\section{" Un partenariat nouveau entre l'assurance maladie et la Mutualité "}

Tel est le vœu du président Nicolas Sarkozy présenté à l'ouverture du $39^{\circ}$ congrès de la Mutualité française, qui s'est tenu à Bordeaux du 4 au 6 juin 2009. Organisation méthodique du déficit de la Sécurité sociale par des exonérations successives de cotisation, réduction sans précédent de la masse salariale: le chef de l'Etat en est convaincu, "les régimes de base ne pourront pas tout financer". Il souhaite donc confier " de nouvelles responsabilités aux organismes complémentaires", confirmant ainsi son intention de parvenir à un désengagement progressif de l'assurance maladie obligatoire, notamment sur les maladies chroniques. Pour JeanPierre Davant, président d'une Mutualité qui a la " volonté de faire bouger les choses » notamment sur ce sujet sensible, "ce que l'assurance maladie obligatoire n'arrive pas à faire, nous pouvons l'assumer ». Le mouvement mutualiste, qui n'est pas rancunier, propose ainsi aux pouvoirs publics d'avoir la possibilité de contractualiser avec des professionnels de santé, afin d'offrir à ses adhérents des filières de santé spécifiques, aux tarifs opposables, sans dépassements d'honoraires. Il n’en fallait pas plus pour que Nicolas Sarkozy applaudisse aux prochaines expérimentations du système. Un congrès qui ouvre un nouveau dossier dans l'histoire des rapports compliqués de la Mutualité et de la Sécurité sociale...

Pour en savoir plus: www.mutualite.fr.

\section{Contrôle des assurances: gare à la banalisation}

"Ouverts mais pas naïfs", tel est le titre de l'éditorial de Jean-Luc de Boissieu, secrétaire 
général du Groupement des entreprises mutuelles d'assurances (Gema) dans sa lettre de mai 2009. En juillet 2008, la loi de modernisation de l'économie a autorisé le gouvernement à rapprocher le contrôle des banques et celui des assurances: "Les mutuelles du Gema se sont montrées ouvertes sur ce sujet, mais elles ne le seront pas au point d'accepter n'importe quelle réforme [, car] il est maintenant question de liquider le contrôle des assurances en le diluant dans les services et dans les méthodes de contrôle de la Banque de France. " Le secrétaire général rappelle donc que les assureurs mutualistes entendent occuper toute leur place dans "le nouveau dispositif de contrôle à construire ". Pour en savoir plus: www.gema.fr. 